\title{
Diffusive Separation of Noble Gases and Noble Gas Abundance Patterns in Sedimentary Rocks T. Torgersen ${ }^{1, *}$, B.M. Kennedy ${ }^{2}$ and M. C. van Soest ${ }^{2}$ \\ ${ }^{1}$ Department of Marine Sciences, University of Connecticut, Groton, CT 06340-6097 \\ ${ }^{2}$ Center for Isotope Geochemistry, Lawrence Berkeley National Laboratory, Berkeley, CA 94720 \\ Corresponding author. Tel: +1-860-405-9094; Fax: +1-860-405-9153. \\ E-mail address: Thomas.Torgersen@uconn.edu
}

\section{Modified 11 June 2004}

ABSTRACT: The mechanisms responsible for noble gas concentrations, abundance patterns, and strong retentivity in sedimentary lithologies remain poorly explained. Diffusion-controlled fractionation of noble gases is modeled and examined as an explanation for the absolute and relative abundances of noble gases observed in sediments. Since the physical properties of the noble gases are strong functions of atomic mass, the individual diffusion coefficients, adsorption coefficients and atomic radii combine to impede heavy noble gas $(\mathrm{Xe})$ diffusion relative to light noble gas ( $\mathrm{Ne})$ diffusion. Filling of lithic grains/half-spaces by diffusive processes thus produces Ne enrichments in the early and middle stages of the filling process with $\mathrm{F}(\mathrm{Ne})$ values similar to that observed in volcanic glasses. Emptying lithic grains/half-spaces produces a Xe-enriched residual in the late (but not final) stages of the process producing $\mathrm{F}(\mathrm{Xe})$ values similar to that observed in shales. 'Exotic ....but unexceptional' shales that exhibit both $\mathrm{F}(\mathrm{Ne})$ and $\mathrm{F}(\mathrm{Xe})$ enrichments can be produced by incomplete emptying followed by incomplete filling. This mechanism is consistent with literature reported noble gas abundance patterns but may still require a separate mechanism for strong retention. A system of labyrinths-with-constrictions and/or C-, Sinanotubes when combined with simple adsorption can result in stronger diffusive separation and nonsteady-state enrichments that persist for longer times. Enhanced adsorption to multiple $\mathrm{C}$ atoms inside C-nanotubes as well as dangling functional groups closing the ends of nanotubes can provide potential mechanisms for 'strong retention'. We need new methods of examining noble gases in rocks to determine the role and function of angstrom-scale structures in both the diffusive enrichment process and the 'strong retention' process for noble gas abundances in terrestrial rocks. 


\section{INTRODUCTION}

A common feature of noble gas abundances in sedimentary rocks is an overall enrichment of heavy noble gases [1-4]. Typical Xe enrichment factors in sedimentary rocks are the order of $F(X e)=10$ 10,000 where:

$$
\mathrm{F}\left[{ }^{\mathrm{i}} \mathrm{Ng}\right]=\frac{\left[{ }^{\mathrm{i}} \mathrm{Ng}\right] /\left[{ }^{36} \mathrm{Ar}\right]_{\text {sample }}}{\left[{ }^{\mathrm{i}} \mathrm{Ng}\right] /\left[{ }^{36} \mathrm{Ar}\right]_{\text {air }}}
$$

and ${ }^{i} \mathrm{Ng}$ denotes any noble gas isotope. Xenon enrichments up to $\mathrm{F}(\mathrm{Xe})=650$ have also been measured in noble gases co-produced with oil and natural gas [5]. The enrichment in both sediments and oil-related fluids have an isotopic composition that is indistinguishable from air and suggests either air and/or airsaturated-water (ASW) as the source. These observed noble gas abundance patterns, which follow a smooth mass dependence with an enrichment of the heavy noble gases, have led to hypotheses that invoke adsorption from air and/or ASW as the responsible process [1-3]. However, adsorption coefficients for noble gases on activated charcoal [6] show only a factor of 30x difference between Xe and Ar at room temperature, thus the large relative Xe enrichments observed in sedimentary rocks seem inconsistent with simple adsorption as the only mechanism. Additionally, laboratory heating experiments suggest that the enriched component is tightly bound [4], counterintuitive to a simple adsorption hypothesis.

Many terrestrial rocks ( half) also contain excess $\mathrm{Ne}$ in conjunction with excess Xe leading Podosek et al [4] to comment that such Ne enrichments “...previously thought to be exotic, must now be considered unexceptional" and to require at least two different processes or events. Typically, $\mathrm{Ne}$ enrichments are smaller than those for $\mathrm{Xe}$, with $\mathrm{F}(\mathrm{Ne})$ ranging from approximately $1-10$ (where $\mathrm{F}(\mathrm{Ne})=$ 0.3 is typical of ASW). Neon enrichments of this magnitude have also been found in oil field gases $[7,8]$. Since simple adsorption is inconsistent with light element enrichment, the higher diffusivity of $\mathrm{Ne}$ relative to the heavy noble gases has led to a diffusive hypothesis as a potential mechanism for Ne enrichment in

rocks [9]. A passive random walk process through a restricted space (interlayer spacing of clays) was suggested [10] that would enhance separation of noble gases, as preferential adsorption would additionally slow the diffusion of one noble gas relative to another. Additionally, a labyrinth-withconstrictions model is described [11] (Figure 1) in which there is active discrimination of large atoms with 
respect to small atoms due to a restricted throat radius on the order of the atomic radius. Neither of these models adequately address the strong retention (release temperatures of $\sim 1000 \mathrm{C}$ ) aspect of noble gases abundances in rocks. The exact mechanism(s) responsible for the large $\mathrm{Xe}$ and $\mathrm{Ne}$ excesses and the strong retention of these excesses over geologic time have remained largely unexplained.

Recent research on carbon nanotubes highlights additional mechanisms that should be considered. It has been found that cutting or heating carbon nanotubes increases adsorption capacity and 'strength' by as much as 10-300x either by allowing direct access to active interior adsorption sites or by releasing dangling functional groups (e.g., $-\mathrm{COOH})$ that may block the entrance to a nanotube [12]. Single walled nanotubes (SWNT) may provide significantly enhanced adsorption when the inside diameter of the nanotube is of the same order as the atomic diameter of the sorbate atom (e.g. Xe), resulting in adsorption (by electron shell interactions) to more than one $C$ atom $[13,14]$. These multiple Cadsorbate 'bonds' may provide an explanation for the high temperatures needed to release (strongly retained) enriched Xe from terrestrial rocks. In meterorites, the elusive Q-phase host for the highly fractionated, heavy noble gas enriched, planetary noble gas component in meteorites may be a carbon nanotube structure [14]. Challa et al [15] propose a quantum sieving mechanism that preferentially adsorbs heavier isotopes relative to lighter ones and have calculated large fractionation factors for ${ }^{1} \mathrm{H}_{2}$ relative to ${ }^{3} \mathrm{H}_{2}$ that show a dependence on nanotube internal diameter. If ${ }^{36} \mathrm{Ar}$ and ${ }^{132} \mathrm{Xe}$ are considered 'isotopes' of a single noble gas element, quantum sieving of noble gases could be expected to produce a very large range in relative enrichments.

The process(es) responsible for strong retention of enriched noble gases (release temperatures of $\sim 1000 \mathrm{C}$ during laboratory experiments) has also not been identified. In addition to enhanced adsorption strength (multiple absorption 'bonds') described above [14], occlusion and trapping have been proposed [16]. If adsorption and enrichment occurs within naturally occurring C-nanotubes in terrestrial rocks, trapping (definition of [16]) may be possible through the creation of dangling functional groups ($\mathrm{COOH}$ ) that would close SWNT [12]. The processes of diagenesis, metamorphism and/or maturation of C-rich (and Si-rich) rocks represent processes by which dangling functional groups might be created in rocks and effectively close the ends of natural nanotubes in a process reverse to that observed [12] with heating experiments. Atwood et al. [17] found highly stable containment of gases within organic 
molecular structures stabilized purely by van der Waals forces. Hohenberg et al [18] examined active capture of physic-sorbed atoms by a growing surface film (crystalline edges and defects are stronger adsorption sites than 'perfect' crystal surfaces) and demonstrated the effectiveness of active and preferential capture of heavy noble gases by Si-smokes deposited in the laboratory. Thus, nanoscale processes offer a distinct mechanism for "tight binding and strong retention", leading to high laboratory release temperatures $(\sim 1000 \mathrm{C})$.

In this paper, we explore a simple 1-D angstrom-scale, half-space model of adsorption and diffusion-controlled transport in a substrate. We also explore a simple labyrinth-with-constrictions model (Figure 1a) and its variant as a carbon (and/or Si) nanotube (Fig 1b) with throat constrictions that induce size-specific diffusive control. We explain both simultaneous and independent enrichments of light and heavy noble gases in terrestrial rocks and evaluate the phenomenon of high temperature release (strong retention) of noble gases in terrestrial rocks.

\section{THE MODEL}

Consider an angstrom-scale half-space of thickness $L$ that experiences a diffusively driven decrease in noble gas concentration due to a changing boundary condition. The dimension $L$ is thus the order of the grain radius, crystallographic layering or nanotube depth. The time required to diffusively

empty the half-space is the order $D_{N g} t / L^{2}$ where $D_{N g}$ is the effective diffusion coefficient of a specific noble gas in the media under investigation and $t$ is real time. Carslaw and Jaeger [19] give the general solution as:

$C=\frac{4 C_{0}}{\pi} \sum_{n=0}^{n=\infty} \frac{-1^{n}}{(2 n+1)} \exp \left[\frac{-D_{N g}(2 n+1)^{2} \pi^{2} t}{4 L^{2}}\right] \cos \frac{(2 n+1) \pi x}{2 L}$

where $C_{o}$ is the initial homogeneous concentration in the half-space/grain. Because terrestrial rock samples are measured in the laboratory as concentrations in the volume defined by $L$ (e.g the grain radius), the solution for the average concentration in the half-space [19] is used:

$$
C_{\text {avg }}=\int_{0}^{L} C \partial x=\frac{8 C_{o}}{\pi^{2}} \sum_{n=0}^{n=\infty} \frac{1}{(2 n+1)^{2}} \exp \left[\frac{-D_{N g}(2 n+1)^{2} \pi^{2} t}{4 L^{2}}\right]
$$

For a half-space filling from an initial concentration of zero, the general solution is: 


$$
C=C-\frac{4 C_{f}}{\pi} \sum_{n=0}^{n=\infty} \frac{-1^{n}}{(2 n+1)} \exp \left[\frac{-D_{N g}(2 n+1)^{2} \pi^{2} t}{4 L^{2}}\right] \cos \frac{(2 n+1) \pi x}{2 L}
$$

and the average concentration in the half-space is:

$$
C_{a v g}=\int_{0}^{L} C \partial x=C_{f}-\frac{8 C_{f}}{\pi^{2}} \sum_{n=0}^{n=\infty} \frac{1}{(2 n+1)^{2}} \exp \left[\frac{-D_{N g}(2 n+1)^{2} \pi^{2} t}{4 L^{2}}\right]
$$

where $\mathrm{C}_{\mathrm{f}}$ is the boundary condition determined concentration to which the half-space fills. In this case, the boundary condition is not the noble gas concentration in the medium external to the half-space (e.g., water) but rather the equilibrium concentration in the half-space to which the external concentration will drive the half-space.

The timescale of the filling and/or emptying process is determined by the effective diffusion coefficient $D_{\mathrm{Ng}}$ that is specific to each noble gas. For a given medium, the diffusivity of each noble gas decreases with increasing molecular weight roughly as a function of $(\mathrm{MW})^{0.5}[20]$. Each noble gas has a specific adsorption coefficient $\left(K_{d}\right)$ that increases with molecular weight roughly as $(M W)^{-1}[20]$. $K_{d}$ determines the ratio of immobile to mobile noble gas atoms [e.g. $\left(\mathrm{mol}_{\mathrm{Ng}} / \mathrm{g}_{\mathrm{solid}}\right) /\left(\mathrm{mol}_{\mathrm{Ng}} / \mathrm{g}_{\mathrm{H} 2 \mathrm{O}}\right)$ where mol is moles and $g$ is grams] and the fraction of each noble gas available for diffusion in the media is governed by the retardation coefficient:

$$
R=1+K_{D} \frac{1-\phi}{\phi} \cdot \frac{\rho_{\text {solid }}}{\rho_{\text {H2O }}}
$$

where $\phi$ is porosity and $\rho$ is density. Thus the effective diffusion rate for each noble gas is:

$$
D_{N g}=\frac{D_{N g}^{\text {molecular }}}{R}
$$

Note that $\mathrm{K}_{d}$ mimics all those mechanisms that enhance diffusion of light noble gases relative to heavy noble gases. (The concept of 'porosity' at the angstrom scale is inappropriate but a parameter with units of porosity is necessary for dimensional consistency, e.g. internal volume of nanotubes/voids per volume crystal matrix).

The effective diffusivity is also affected by the throat size in the labyrinth-with-constrictions model or the electron shell interactions with the carbon (or Si) atoms of a nanotube. We consider a very simple mechanism of throat/nanotube interaction. The probability of a noble gas atom with an atomic radius of 
$r_{\mathrm{Ng}}$ passing through a throat is a function of the throat radius, $r_{t}$. For the condition where $r_{t}<r_{N g}$, the atom must deform its electron cloud and slip (instantaneously) through a narrow but short throat (simplistic quantum tunneling). To conserve volume, the atom must take on an ellipsoidal shape for a short time such that:

$\mathrm{ABC} / \mathrm{r}_{\mathrm{Ng}}{ }^{3}=1$

where $A, B, C$ are the lengths of the major and minor axes. As two axes must shorten simultaneously, the probability is simply estimated to be (see Figure 2):

$\mathrm{P}=\mathrm{r}_{\mathrm{t}}^{2} / \mathrm{r}_{\mathrm{Ng}}^{2}$ for $\mathrm{r}_{\mathrm{t}}<\mathrm{r}_{\mathrm{a}} \quad$ and $\mathrm{P}=1$ for $\mathrm{r}_{\mathrm{t}}>\mathrm{r}_{\mathrm{Ng}}$

The exact formulation of the probability is not critical, since only the relative effects of diffusion are being explored. Thus, the net effect of adsorption and throat diameter is to decrease the effective diffusivity of high molecular weight noble gases relative to low molecular weight noble gases $\left(D_{N g}\right.$ decreases with increasing MW; $\mathrm{K}_{\mathrm{d}}$ increases with increasing MW; and atomic radius increases with increasing MW).

Specific information with regard to the absolute and relative adsorption coefficients of noble gases from air saturated aqueous media onto sedimentary materials is limited $[6,21,22]$ and not directly applicable. Hence, to scale relative adsorption coefficients, we have chosen adsorption coefficients for noble gases on activated charcoal at $273 \mathrm{C}$ [6]. The relative adsorption coefficients are tied to an absolute scale using the adsorption data for $\mathrm{Kr}$ on shales [22]. In general, $\mathrm{He}$ and $\mathrm{Ne}$ exhibit little or no adsorption and the ratio of Xe adsorption to Ar adsorption is of the order 25. Thus, the hypothetical distribution coefficients shown in Table 1 (which scales the Ar:Kr and Ar:Xe difference by the values for activated charcoal [6] and the absolute magnitude by an assumed $\mathrm{K}_{d}{ }^{\mathrm{Xe}} \bullet \rho_{\text {rock }} / \rho_{\mathrm{H} 2 \mathrm{O}}=50$ [22]) provide a conservative estimate of the adsorption coefficients for the noble gas suite. With an assumed porosity of $\sim 0.01$ and the above estimates, relative retardation coefficients are calculated with respect to $D_{\mathrm{Ne}}=1$ and are given in Table 1. Table 2 summarizes the range of conditions used in this work.

\subsection{The Emptying Substrate}

The initial condition for noble gases in an absorbant substrate is chosen to be $F(\mathrm{Ne})=0.1, F(\mathrm{Ar})=1$, $F(K r)=13$ and $F(X e)=186$ corresponding to the $\mathrm{KH}-77-1-7$ seafloor sediment [4]. This is a very young ocean sediment that we consider (arbitrarily) to be the passive, adsorption-only endmember that has not yet been affected by diffusive mechanisms for enrichment/depletion. (In Figure 5a, KH-77-1-7 shows the 
greatest relative and absolute enrichments of Xe among the young ocean sediments). Furthermore, our choice of $\mathrm{KH}-77-1-7$ can be simplistically justified with $F(\mathrm{Ne})_{\text {seawater }}=0.3, F(\mathrm{Xe})_{\text {seawater }}=4$, and $\mathrm{K}_{\mathrm{d}}{ }^{\mathrm{Xe}} \rho_{\text {rock }} / \rho_{\mathrm{H} 2 \mathrm{O}}=50$ and $\mathrm{K}_{d}^{\mathrm{Ar}} \rho_{\text {rock }} / \rho_{\mathrm{H} 2 \mathrm{O}}=2$ leading to an initial sediment composition of: $\mathrm{F}(\mathrm{Xe})_{\text {sediments }}=$ $4^{*} 50 / 2=$ order 100 and $F(\mathrm{Ne})_{\text {sediments }}=0.3 / 2=$ order 0.1 .

Figure 3 shows the general results for emptying. (For simplicity, Argon and Krypton have not been included in Figure $3 a(4 a)$.) Approximately $90 \%$ of any noble gas will be lost after a time $D_{\mathrm{Ng}} t / L^{2}=1$ and close to $100 \%$ will be lost after $D_{N g} t / L^{2}=3$, where $D_{N g}$ is the effective diffusivity of the noble gas in question. Thus the slab will empty in the order $\mathrm{Ne}, \mathrm{Ar}, \mathrm{Kr}, \mathrm{Xe}$. In order to relate one noble gas to another noble gas, equations $(3,5)$ are solved for each noble gas in the range of at least $D_{N g} t / L^{2}=0.01$ to $D_{N g} t / L^{2}=$ 3 , and each noble gas specific time is then converted to dimensionless "neon time" using the relation: $D_{N e} t / L^{2}=D_{N g} t / L^{2 *} D_{N e} / D_{N g}$. From Figure 3a, it is apparent that the throat restriction chosen has a small effect compared to $\mathrm{K}_{\mathrm{d}}$.

The effects of slab emptying on relative abundances is shown in Figure $3 \mathrm{~b}$ (for simplicity, $\mathrm{F}(\mathrm{Kr})$ results have been omitted from Figure $3 b(4 b))$, from which it is evident that (1) the long timescale residual will be enriched in Xe (because Ar leaves the substrate faster) and (2) the short timescale residual will be depleted in $\mathrm{Ne}$ (because $\mathrm{Ar}$ is retained longer). When the range of effective diffusivities is large $\left(K_{d}=0,2,11,50\right), F(\mathrm{Ne})$ depletions can occur well before enrichments in $F(X e)$. When the range of effective diffusivities is small $\left(K_{d}=0,0,0,0\right)$, depletions in $F(N e)$ are followed closely in time by enrichments in $F(X e)$. Secondly, near the end of emptying, the potential enrichment of $F(X e)$ is very large no matter what the effective diffusivities and $F(X e)$ values can be achieved that are consistent with observations in sedimentary rocks (e.g., [2,4]) and fluids [5,7], e.g. $F(X e)=500-10,000$. Pinti et al. [23] produced a similar model as an explanation for preferential diffusive loss of $\mathrm{Ne}$ from pumice to vesicles using relative (not ablsolute) diffusion rates.

\subsection{The Filling Substrate}

For an idealized absorbant substrate that initially does not contain any noble gases, a final condition is chosen to be $F(\mathrm{Ne})=0.1, F(\mathrm{Kr})=13$ and $F(\mathrm{Xe})=186$ corresponding to the $\mathrm{KH}-77-1-7$ seafloor sediment sample [4]. The results are shown in Figure 4. The same generalities apply ( $\mathrm{Ne}$ is transported faster than $\mathrm{Ar}$, etc.) but the effect on the noble gas enrichment patterns (Figure $4 \mathrm{~b}$ ) requires some explanation. 
For short times, the enrichment in $\mathrm{F}(\mathrm{Ne})$ occurs because Ne enters the system more readily than $\mathrm{Ar}$. However, the initial $F(\mathrm{Xe})$ (at short times) is not significantly different from the boundary condition. This is because the outside surface of the half-space quickly acquires $\operatorname{Ar}$ and $\mathrm{Xe}$, and an $\mathrm{F}(\mathrm{Xe})$ value that is in equilibrium with the external media, while the rest of the half space remains largely empty. Thus, this short time $F(X e)$ reflects the surface-only concentrations of Xe and Ar. As Ar diffuses into the system faster than $\mathrm{Xe}$, there is a resulting depletion in $\mathrm{F}(\mathrm{Xe})$ that is slowly erased as the half-space is eventually filled with $X e$ and $F(X e)$ of the slab achieves the boundary-imposed final condition for $F(X e)$. Given the small surface-only concentrations of Ar and Xe that contribute to the observation of "no initial depletion", such small concentrations of noble gas might not be visible above typical procedural blanks encountered in the mass spectrometer measurements.

\subsection{Model Summary}

It is concluded that (1) Xe enrichments of the order $F(X e)=10^{3}-10^{7}$ can be achieved by diffusive separation in the unsteady state emptying of a substrate, and (2) Ne enrichments of the order $F(\mathrm{Ne})=1-5$ can be achieved by diffusive separation in the unsteady state filling of a substrate. These noble gas enrichments are well above expectations based on simple adsorption phenomenon (e.g., $\mathrm{F}(\mathrm{Ne})=0.1$, $F(K r)=13$ and $F(X e)=186$ corresponding to $\mathrm{KH}-77-1-7$ seafloor sediment [4]), and can be achieved on

timescales of the order $\mathrm{D}_{\mathrm{Ng}} \mathrm{tL}^{-2}=1$ for both molecular diffusivity-only and molecular diffusivity inhibited by adsorption/retardation and/or throat constrictions. The enrichment in $F(X e)$ persists until $\mathrm{Xe}$ is diffusively lost from the substrate $\left(D_{x_{e}} t^{-2}=3\right)$ and is driven by the faster loss of Ar from the substrate. The enrichment in $F(\mathrm{Ne})$ is created at short times by filling a substrate with $\mathrm{Ne}$ filling faster than $\mathrm{Ar}$. The $\mathrm{F}(\mathrm{Ne})$ enrichment is lost on the timescale of $D_{A r} t^{-2}=3$ as the substrate is filled with Ar. These noble gas enrichments occur at the expense of concentration and the magnitude of observable enrichments is critically dependent upon the precision to which low noble gas concentrations can be measured. Simple adsorption retardation and throat constriction enhance the diffusive separation of noble gases but does not change the above conclusions (it does change $D_{\mathrm{Ng}}$ ). However, enhanced diffusive separation of noble gases increases the time interval over which the (unsteady-state) $F(\mathrm{Ne})$ and $F(X e)$ enrichments can be found in grain/half-spaces and might therefore be sampled/measured. This increases the probability that $F(\mathrm{Ne})$ and $F(\mathrm{Xe})$ enrichments will be observed in terrestrial rocks. 


\subsection{Altering Boundary Conditions}

Boundary condition changes can be generated inside or outside the rock. Thus natural terrestrial processes should lead to diffusion-controlled conditions driven by chemical activity gradients (functions of concentration, temperature, pressure and solubility) that allow the filling and emptying that creates noble gas enrichment and depletions of the order seen in terrestrial rocks. The following processes might contribute to an emptying grain/half-space and an enrichment in $F(X e)$.

1. Sedimentary shales could originate from ocean sediments that initially contain an adsorbed component at deep ocean temperatures $(\sim 0-2 C)$. Subsequent heating due to burial and the geothermal gradient will decrease the effective adsorption coefficients leading to $F(X e)$ enrichments caused by faster diffusive loss of light noble gases. Since the sediments that have been measured $[4,9,24]$ all show a Xe enrichment (Figure 5), we believe that sediments are deposited with an adsorbed component in noble gases and enhance their $F(X e)$ by noble gas emptying rather than by strong preferential adsorption.

2. Diagenesis/maturation of lithified rock could generate a surfactant that would reduce the adsorption coefficient. A surfactant would cause an emptying of the rock and might explain the high values of $F(X e)$ observed in oil field fluids [5]. Changes in surface mineral properties within a lithifying rock could also change the adsorption properties and allow emptying.

3. Emptying could be driven by circulation of degassed waters having low noble gas concentrations. Such might occur during hydrothermal circulation and/or methanogenesis where bubble ebullition effectively strips gases from the surrounding waters.

4. Simple compaction of sediments (lithification) would reduce the porosity and have the same affect as increasing the distribution coefficient. Since retardation also applies to advective water losses, compaction could also lead to an emptying system and a relative increase in $F(X e)$.

The filling of an adsorbant substrate could be driven by the reverse of the conditions noted above (e.g.) (1) a decrease in temperature that effectively raises the adsorption coefficient, (2) the loss of surfactant and/or mineral surface properties that leads to an increase in the adsorption coefficient, and (3) an unroofing that would lead to flow of concentrated fluids into the rock and an increase in porosity. It should be noted that most $F(\mathrm{Ne})$ enrichments occur with some degree of $F(X e)$ enrichment (Figure 5). 
Thus most $\mathrm{F}(\mathrm{Ne})$ enriched systems are an incomplete emptying followed by an incomplete filling of the same system.

\subsection{Strong Retention: Trapping, Very Strong Adsorption or Artifact}

Laboratory observations indicate that release of the enriched/depleted noble gas components from sedimentary rocks requires heating to high temperatures ( 1000C, [4]). This observation of "strong retention" has lead to several hypotheses that invoke trapping, occlusion [16], very strong adsorption or active capture [18]. Strong retention of noble gas components could also be the result of additional nanotube mechanisms $[12,17]$. However, to what extent is a separate mechanism necessary?

The $1000 \mathrm{C}$ temperatures required for release of the enriched noble gas component suggests effective diffusivities on the order of $10^{-19} \mathrm{~cm}^{2} \mathrm{~s}^{-1}$ [11]. Solomon et al. [25], give values for the diffusion of helium in 'aquifer solids' of the order $10^{-17} \mathrm{~cm}^{2} \mathrm{~s}^{-1}$. For $1 \mathrm{~mm}$ grains typical for mass spectrometric analysis, the filling/emptying time is predicted to be $\left(t=L^{2} D_{N g}{ }^{-1}\right) 30 \mathrm{Myr}$ to $3000 \mathrm{Myr}$. Overnight pumping at $100 \mathrm{C}$ is not going to remove a diffusion implanted (over geologic time) noble gas signature. Even at $600 \mathrm{C}$, diffusion coefficients are of the order $10^{-10} \mathrm{~cm}^{2} \mathrm{~s}^{-1}$ [25] and would require 3 years to empty the grain. However, on a geologic timescale there is more than adequate time to create changing boundary conditions that drive filling/emptying and Ne-, Xe-enrichment, as well as, a high probability ( half, [4]) that incomplete emptying will be followed by incomplete filling and result in lithologies enriched in both $\mathrm{F}(\mathrm{Ne})$ and $F(X e)$.

Do we need the additional strong retention mechanisms that could potentially be supplied by e.g. nanotubes [11-18]? Certainly, geologic time scales are available to create enrichments/depletions observed in the rock types that have been investigated [1-4, 9, 10, 21-28]. However, the noble gas enrichments observed in fluids $[5,7]$ create a problem. The timescale of fluid flow for many aquifer/oil systems is of the order $200,000 y r s[26,27]$. This suggests that the timescale for exchange between rock and liquid must be of the order $<10^{6} \mathrm{yrs}$. The inferred relation between the liquid and the solid $[5,7]$ suggests that some type of 'switch' is necessary to explain both the measurements in the solid phase and the measurements in the liquid phase. Shorter time scale diffusion processes $(\mathrm{L}$ small and therefore shorter time) coupled with the opening/closing of e.g. nanotubes (related to maturation and oil expulsion) may be operational and should be investigated. 
If the probability for passing through a throat restriction is more important than has been simplistically modeled here, or enhanced adsorption through tuning of a nanotube diameter to the adsorbed noble gas (allowing adsorption with multiple $C$ atoms) is significant [14], then the time required to achieve significant enrichment is shortened. If dangling functional groups opening/closing nanotubes [12], and organic supramolecular containment stabilized by van der Waals forces [17] are viable mechanisms in nature, then short time scale release/accumulation can be accompanied by long time scale containment. It is likely that occlusion [16] and active capture [18] can be eliminated as mechanisms as they involve surface features not amenable to diffusive enrichment processes. However, growing surfaces that close labyrinth-with-constrictions pores and/or SWNT may act similarly to 'dangling functional groups' and 'strongly retain' noble gases. We need new methods of examining noble gases in rocks to determine the role and function of angstrom-scale structures in the diffusive enrichment process and the 'strong retention' process for noble gas abundances in terrestrial rocks.

\section{COMPARISON OF MODEL CONCEPTS AND LITERATURE DATA}

To evaluate the diffusive separation model, the noble gas signatures of sediments are compared to the shales to which they often transform. Figure 5a shows the relative noble gas patterns of young ocean sediments $[4,9,24]$ (recovered as piston cores from the ocean floor and therefore on the order of $<100 \mathrm{Myr}$ ). All sediments show a pattern exhibiting progressive enrichment with increasing atomic mass. It is important to note that $\mathrm{F}(\mathrm{Ng})$ values are with respect to air while the noble gas source is more likely air-saturated-seawater: $\mathrm{F}(\mathrm{Ne}) \sim 0.28$ and $\mathrm{F}(\mathrm{Xe}) \sim 3.5$ (represented by the dashed gray lines in Figure 5). With respect to air-saturated-seawater (dashed gray line), all of the samples indicate Ne depletion and $\mathrm{Kr}$-Xe enrichment. The samples also exhibit a tight range in $\mathrm{F}(\mathrm{Ng})$ values (Figure $5 \mathrm{a}$ ) and concentrations (Figure 5b). The tight compositional ranges are consistent with a simple and universal acquisition process with little or no subsequent processing (gas loss/gain) effecting individual samples.

Figure $5 c$ shows the noble gas pattern of shales reported in the literature $[4,10,28$; one Pleistocene claystone [10] and kerogen fractions [28] have been omitted]. It is apparent that $\mathrm{F}(\mathrm{Ne})$ enriched shales are quite common (Ne enrichments "previously thought to be exotic, must now be considered unexceptional" [4]). In the sediment-to-shale metamorphic sequence, most shales (Figure 5d) are more concentrated in $\mathrm{Ne}$ and are less concentrated in Xe (albeit with considerable variability) than the 
potential precursor sediments (Figure 5b). For the diffusive separation to provide a viable explanation, sediments must empty a significant fraction of the noble gases during the diagenesis/lithification process to enrich the $F(X e)$ signal after which some shales begin a filling process that causes the enrichment in $\mathrm{F}(\mathrm{Ne})$. Critical experiments detailing the metamorphic history of sedimentary lithologies combined with noble gas studies should prove interesting.

Figure 5e shows the noble gas enrichment pattern of Mesozoic cherts $[29,30]$. The silica of these sediments includes both biogenic and diagenic components and some samples have a hydrothermal component. For the young ocean sediment-to-chert metamorphic sequence, there appears to be a relative decrease in the enrichment of $F(X e)$ and a relative increase in $F(\mathrm{Ne})$ (Figure 5e). Secondly, the concentration of $\mathrm{Ar}$ and $\mathrm{Xe}$ in cherts (Figure $5 \mathrm{f}$ ) is significantly lower than that found in other sediments (Figure 5b), although comparable with respect to $\mathrm{Ne}$. If sediments evolve to cherts, then the sediment system must first loose $\mathrm{Ar}$ and $\mathrm{Xe}$ (and therefore $\mathrm{Ne}$ as well) leaving it with a small residual $\mathrm{Xe}$ and $\mathrm{F}(\mathrm{Xe})$ enrichment. If the system then refilled, strong $F(\mathrm{Ne})$ enrichment would be observed and decreases in $F(X e)$ could be expected if these cherts have filled through 'Ar time' $\left(D_{A r} t^{-2}=1\right)$ but have not yet entered 'Xe time' $\left(D_{\text {Xe }} \mathrm{tL}^{-2}=1\right)$. Alternatively, young sediment-to-chert conversion could represent a non-continuous sequence, in which case the noble gas patterns and concentrations may reflect initial conditions and not an evolved composition. The relative noble gas compositions in the cherts are not all that different from air-saturated-water, consistent with bulk "trapping" from water during formation.

The noble gas abundances in obsidians and glass [31] are of interest because their high temperature of formation suggests little adsorption and small initial concentrations. Upon exposure to air/water and subsequent cooling, such rocks are susceptible to filling and diffusion controlled $\mathrm{F}(\mathrm{Ne})$ enrichment. Figure $6 a, b$ show that most volcanic glass samples have strong $F(\mathrm{Ne})$ enrichment patterns. In comparison to sediments (Figure 5b), the concentrations of $\mathrm{Ar}$ and Xe are 10-100x less in volcanic glasses while $\mathrm{Ne}$ is $10 x$ higher. As argued by Matsuda et al. [31], this strongly supports diffusively controlled filling of volcanic rocks as the primary control on the $F(\mathrm{Ne})$ pattern in these glasses.

\section{CONCLUSIONS}

Noble gas abundances and their corresponding enrichment factors observed in sedimentary rocks and volcanic glasses can be explained by diffusion-controlled noble gas separation. The diffusion 
coefficients, adsorption coefficients and atomic radii of the noble gases, being strong functions of atomic mass, mutually interact to enhance noble gas fractionation: Xe diffuses the slowest, Ne the fastest (excluding $\mathrm{He}$ from the discussion). Incomplete diffusive filling of lithic grains produces $\mathrm{F}(\mathrm{Ne})$ enrichment, as notably exhibited by volcanic glasses (Figure 6). Incomplete emptying of lithic grains produces an $\mathrm{F}(\mathrm{Xe})$ enriched residual, as exhibited by shales (Figure 5c,d). The 'exotic ...but unexceptional' shales that exhibit both $F(\mathrm{Ne})$ and $F(X e)$ enrichments are likely the result of an incomplete emptying of lithic grains followed by an incomplete filling in the sediment-to-shale metamorphic process

The timescales for diffusive enrichment/depletion and the identification of enriched noble gas signals in both the solid phase (sediments and shales) and the fluid phase (oil and water samples) suggests that an open/closed 'switch' may still be necessary for strong retention and suggests a potential role for nanoscale closure mechanisms. The potential role of $\mathrm{C}$ - and/or Si-nanotubes as a mechanism for diffusive separation as well as dangling functional groups and enhanced adsorption as a means for strong retention of noble gases merits further investigation. We need more and better data along with new methods of examining noble gases in sedimentary lithologies to determine the role and function of angstrom-scale structures in the diffusive enrichment and the 'strong retention' processes effecting noble gas abundances. It is also clear that sample handling and sample preparation are a part of the noble gas metamorphic history of the rock and studies similar to that of Bernatowicz et al. [10] may improve our ability to distinguish between those signals induced by laboratory 'metamorphism' from those induced by natural metamorphism. Interesting possibilities present themselves for the interpretation of "noble gas metamorphism" when combined with radiogenic and nucleogenic noble gas species that introduce a unique time and chemical (parent/daughter ratio) dependence. Given that many rocks have independent thermochronologies and coupled noble gas histories, the acquisition of a noble gas signature by shale, rock, gas, oil and or water by extensive rock/water interaction will likely be distinctive and provide a unique source marker.

\section{ACKNOWLEDGEMENTS}

This work was supported in part by the Director, Office of Energy Research, Office of Basic Energy Sciences, Engineering and Geosciences Division of the US Department of energy under contracts DE-AC03-76SF00098 and DE-FG02-95ER14528. TT wishes to acknowledge the suggestions of Mike 
Celia in the formulation of this problem and National Science Foundation for support during the final preparation of this manuscript. The authors would also like to acknowledge Mark Kurz and an anonymous reviewer for their constructive comments.

\section{REFERENCES}

[1] R.A. Canalas, E.C. Alexander, O.K. Manuel, Terrestrial abundance of noble gases, J. Geophys. Res. 73 (1968) 3331-3334.

[2] F.P. Fanale, W.A. Canon, Physical adsorption of rare gas on terrigenous sediments, Earth Planet. Sci. Lett. 11 (1971) 362-386.

[3] F.P. Fanale, W.A. Canon, Origin of planetary primordial rare gas: the possible role of adsorption, Geochim. Cosmochim. Acta 36 (1972) 319-328.

[4] F.A. Podosek, M. Honda, M Ozima, Sedimentary Noble Gases, Geochim. Cosmochim. Acta 44 (1980) 1875-1884.

[5] T. Torgersen, B.M. Kennedy, Air-Xe enrichments in Oil Field Gases and the Influence of Water during Oil Migration and Storage, Earth Planet Sci. Lett. 167 (1999), 239-253.

[6] S. Dushman, Scientific foundations of Vacuum Technique, John Wiley and Sons, NY, 1957, 806pp.

[7] B.M. Kennedy, T. Torgersen, M. van Soest, Multiple atmospheric noble as components in hydrocarbon reservoirs: a study of the northwest shelf, Delaware Basin, SE New Mexico, Geochim. Cosmochim. Acta 66 (2002) 2807-2822.

[8] A.P. Battani, P. Sarda, A. Prinzhofer, Basin scale natural gas source, migration and trapping by noble gases and major elements: The Pakastani Indus Basin, Earth Planet. Sci. Lett. 181 (2000) 229249.

[9] J. Matsuda, K. Matsubara, Noble gases in silica and their implication for the terrestrial 'missing Xe. Geophys Res. Lett. 16 (1989) 81-84.

[10] T. Bernatowicz, F.A. Podosek, M. Honda, F.E. Kramer, The atmospheric inventory of Xenon on noble gases in shales: the plastic bag experiment, J. Geophys Res. 89 (1984) 4597-4611.

[11] J.F. Wacker, M.G. Zadnik, E. Anders, Laboratory simulation of meteoritic noble gases I sorption of xenon on carbon: trapping experiments, Geochim. Cosmochim. Acta 49 (1985) 1035-1048.

[12] A. Kuznetsova, J.T. Yates, J. Liu, R.E. Smalley, Physical observation of xenon in open single walled carbon nanotubes: observation of a quasi-one-dimensional confined Xe phase, J. Chem. Phys. 112 (2000) 9590-9598 [S0021-9606(00)71313-4].

[13] V. Simonyan, J.K. Johnson, A. Kuznetsova, J.T. Yates, Molecular simulation of xenon adsorption on single walled carbon nanotubes, J. Chem. Phys. 114(2001) 4180-4185 [DOI:10.1063/1.1344234].

[14] R.D. Vis., A. Mrowiec, P.J. Kooyman, K. Matsubara, D. Heymann, Microscopic search for the carrier phase $Q$ of the trapped planetary noble gases in allende, Leoville and vigarano, Meteoritics and Planet. Sci. 37(2002)1391-1399.

[15] S.R. Challa, D.S. Scholl, J.K. Johnson, Light isotope separation in carbon nanotubes through quantum molecular sieving, Phys. Rev. B 63 (2001) 245419 (9 pages), [DOI10.1103/PhysRevB.63.245419].

[16] J. Yang, R.S. Lewis, E. Anders, Sorption of noble gases by solids with reference to meteorites I magnetite and carbon, Geochim. Cosmochim. Acta 46 (1982) 841-860.

[17] J.L. Atwood, L.J. Barbour, A. Jerga, Storage of methane and freon by interstitial van der Walls confinement, Science 296 (2002) 2367- 2369.

[18] C.M. Hohenberg, N. Thonnard, A. Meshik, Active capture and anomalous adsorption: new mechanisms for the incorporation of heavy noble gases, Meteoritics and Planet. Sci. 37 (2002) 257-267.

[19] H.S. Carslaw, J.C. Jaeger, Conduction of Heat in Solids, Claredon Press, Oxford, 1959, 510pp.

[20] R.B. Bird, W.E. Stewart, E.N. Lightfoot, Transport Phenomena, John Wiley and Sons, NY, 1960, 780pp.

[21] J. Yang, E. Anders, Sorption of noble gases by solids with reference to meteorites II: chromite and carbon, Geochim. Cosmochim. Acta 46 (1982) 861-875. 
[22] F.A. Podosek, T.J. Bernatowic, F.E. Kramer, Adsorption of Xenon and Krypton on shales, Geochim. Cosmochim. Acta 45 (1981)2401-2415.

[23] D.L. Pinti, N. Wada, and J.-I. Matsuda, Neon excess in pumice: volcanological implications, J. Volcanol. Geotherm. Res. 88 (1999) 279-289.

[24] J. Matsuda, K. Nagao, Noble gas abundances in deep-sea sediment core from eastern equatorial Pacific, Geochem. Jour. 20 (1986) 71-80.

[25] D.K. Solomon, A. Hunt, and R.J. Poreda, Source of radiogenic helium-4 in shallow aquifers: implications for dating young groundwater, Water Resources Res. 32 (1996) 1805-1813.

[26] G. Garven, The role of regional fluid flow in the genesis of the Pine Point Deposit, western Canada sedimentary basin, Econ. Geol. 80 (1985) 307-324.

[27] G. Garven, A hyrolgeologic model for the formation of giant oil sands deposits of the western Canada sedimentary basin, Amer. Jour. Sci. 289 (1989) 105-166.

[28] U.Frick, S. Chang, Ancient carbon and noble gas fractionation, Proc. Lunar Science Conf. $8^{\text {th }}(1977)$ 263-272.

[29] K.Matsubara, J. Matsuda, K. Nagao, I. Kita, Taguchi, Xe in amorphous silica: a new thermometer in geothermal systems, Geophys Res Lett 15 (1988) 657-660.

[30] K. Matsubara, J. Matsuda, R. Sugasaki, Noble gases in Mesozoic cherts from the USA and Japan, Chemical Geol. 86 (1991): 287-293.

[31] J. Matsuda, K. Matsubara, H. Yajima, K. Yamamoto, Anomalous Ne enrichment in obsidians and Darwin Glass: diffusion of noble gases in silica rich glasses, Geochim. Cosmochim. Acta 53 (1989) 3025-3033. 
Table 1: Measurements of the distribution coefficient of noble gases on charcoal at room temperature [6] as an analog for the adsorption of noble gases on organic rich shales in the terrestrial environment. Given the adsorption coefficient of $\mathrm{Xe}$ on natural $\mathrm{C}$-rich rock $\left(\mathrm{K}_{d}{ }^{\mathrm{Xe}}=50\right)$ the natural rock adsorption coefficients were scaled from charcoal data. Assuming a porosity of $\sim 0.01$, the $\mathrm{K}_{d}$ are combined to calculate a retardation coefficient, $R$. For ease of comparison ( $1 /$ retardation coefficient=1/R) is also given that indicates the relative effect on the diffusive separation of noble gases.

\begin{tabular}{|c|cccc|}
\hline & $\begin{array}{c}\text { adsorption on charcoal } \\
\mathrm{cm}^{3} \mathrm{Ng} / \mathrm{mm}-\mathrm{gC}\end{array}$ & $\mathrm{K}_{\mathrm{d}} \rho_{\text {rock }} / \rho_{\mathrm{H} 2 \mathrm{O}}$ & $1 / \mathrm{R}$ & $\mathrm{R}$ \\
\hline $\mathrm{Ne}$ & 0 & 0 & 1 & 1 \\
$\mathrm{Ar}$ & 0.058 & 2 & 0.004975 & 201 \\
$\mathrm{Kr}$ & 0.34 & 11 & 0.000908 & 1101 \\
$\mathrm{Xe}$ & 1.583 & 50 & 0.0002 & 5001 \\
& \multicolumn{4}{|c}{ assuming $\mathrm{K}_{\mathrm{d}}{ }^{\mathrm{Xe}} \rho_{\text {rock }} / \rho_{\mathrm{H} 2 \mathrm{O}}=50$} \\
\hline
\end{tabular}

Table 2: Illustrative values of the effective diffusion coefficients of noble gases relative to a Ne effective diffusivity $D_{\mathrm{Ne}}=1$ and a square root of molecular weight dependence of diffusivity. $K_{d}$ and throat diameters indicated are used to illustrate a conservative impact of those parameters on diffusion separation. The atomic radii (angstroms) are $\mathrm{Ne}=0.6, \mathrm{Ar}=0.8, \mathrm{Kr}=1.1$, and $\mathrm{Xe}=1.3$

\begin{tabular}{|c|c|c|c|}
\hline \multirow[t]{3}{*}{$\begin{array}{c}\text { Throat } \\
\text { Angstroms } \\
\end{array}$} & \multicolumn{3}{|c|}{$\begin{array}{l}\text { Effective Diffusion Coefficient } \\
\text { Labyrinth-with-constrictions/nanotube Model }\end{array}$} \\
\hline & $\mathrm{Ne}$ & $\overline{A r}$ & $\overline{\mathrm{Xe}}$ \\
\hline & $\mathrm{K}_{\mathrm{d}} \rho_{\text {rock }} / \rho_{\mathrm{H} 2 \mathrm{O}}=0$ & $\mathrm{~K}_{\mathrm{d}} \rho_{\text {rock }} / \rho_{\mathrm{H} 2 \mathrm{O}}=2$ & $\mathrm{~K}_{\mathrm{d}} \rho_{\text {rock }} / \rho_{\mathrm{H} 2 \mathrm{O}}=50$ \\
\hline 0.6 & 1 & 0.002188 & 1.75E-05 \\
\hline 0.8 & 1 & 0.003889 & $3.12 \mathrm{E}-05$ \\
\hline \multirow[t]{2}{*}{1.3} & 1 & 0.003889 & 8.23E-05 \\
\hline & $\mathrm{K}_{\mathrm{d}} \rho_{\text {rock }} / \rho_{\mathrm{H} 2 \mathrm{O}}=0$ & $\mathrm{~K}_{\mathrm{d}} \rho_{\text {rock }} / \rho_{\mathrm{H} 2 \mathrm{O}}=0$ & $\mathrm{~K}_{\mathrm{d}} \rho_{\text {rock }} / \rho_{\mathrm{H} 2 \mathrm{O}}=0$ \\
\hline 0.6 & 1 & 0.439 & 0.0876 \\
\hline 0.8 & 1 & 0.781 & 0.155 \\
\hline 1.3 & 1 & 0.781 & 0.4113 \\
\hline
\end{tabular}


Figure 1: (A) The labyrinth-with-constrictions model (after [11]) and (B) the single walled nanotube modification used to examine diffusive separation of noble gases in terrestrial rocks. Note that the throat size (nanotube diameter) will allow the smaller atom to pass easily but that the larger atom must alter the shape of its electron cloud for a time long enough to pass through the throat constriction. The difference between a labyrinth-with-constriction (Figure 1a) and a nanotube (Fig 1b) is the lack of interior nodes with larger diameters in the latter.

Figure 2: The probability that a noble gas will pass through a throat constriction based on conservation of volume of the atomic electron cloud (eqn 8). For each noble gas there is a distinct dependency as a function of throat size up to the atomic radii after which the probability is one. The atomic radii (angstroms) are $\mathrm{Ne}=0.6, \mathrm{Ar}=0.8, \mathrm{Kr}=1.1$, and $\mathrm{Xe}=1.3$ [6].

Figure 3: (A) The relative rates by which a half-space of thickness $\mathrm{L}$ is emptied of $\mathrm{Ne}$ (solid gray line, left axis) and Xe (various conditions; right axis) by a change in the boundary condition. Note that $D_{\mathrm{Ng}} t / \mathrm{L}^{2}=3$ is a characteristic time for complete loss of noble gas and $D_{N g} t / L^{2}=1$ represents the timescale over which the most rapid change is occurring. The figure is plotted with relative concentrations for the conditions given in Table 2. Note the relative degree of separation that results from retardation and throat constrictions. Emptying is driven by a boundary condition is not the noble gas concentration in the medium external to the half-space (e.g., water) but rather the equilibrium concentration in the half-space to which the external concentration will drive the half-space. (B) The relative enrichment/depletion of noble gases as a function of time and effective diffusivities for the emptying slab. For minimal (maximal) differences in the effective diffusivities, note the short (long) temporal separation for the depletions in $\mathrm{Ne}$ and enrichments in Xe.

Figure 4: (A) The relative rates by which a half-space of thickness $L$ is filled with $\mathrm{Ne}$ (solid gray line; left axis) and Xe (various conditions; right axis) by a change in the boundary condition. Note that $D_{\mathrm{Ng}} t / L^{2}=3$ is a characteristic time for the complete filling loss of noble gas and that $D_{\mathrm{Ng}} t / \mathrm{L}^{2}=1$ represents the timescale over which the most rapid change is occurring. The figure is plotted with relative concentrations for the conditions given in Table 2. Note the relative degree of separation that results from retardation and throat constrictions. The figure is plotted with relative concentrations for the conditions given in Table 2. Note the relative degree of separation that results from retardation, and throat constrictions. Emptying is driven by a boundary condition is not the noble gas concentration in the medium external to the half-space (e.g., water) but rather the equilibrium concentration in the half-space to which the external concentration will drive the half-space. (B) The relative enrichment/depletion of noble gases as a function of time and effective diffusivities for the filling slab. For minimal (maximal) differences in the effective diffusivities, note the short (long) temporal separation for the enrichments in $\mathrm{Ne}$ and depletions in $\mathrm{Xe}$.

Figure 5: Noble gas abundance patterns and noble gas concentrations reported in the literature $[4,9,10,24,28,30]$. Only whole rock analyses are plotted. For all figures, $\mathrm{KH}-77-1-7$ is plotted as the solid gray line and the dashed gray line indicates the air-saturated-seawater with $10 \%$ porosity. (a) The enrichment pattern of Noble gases in young sediments (core recoveries and therefore of the order $<10^{8}$ years reported by $[4,9,24]$. $\mathrm{KH}-77-1-7$ shows the greatest relative $\mathrm{F}(\mathrm{Xe})$ enrichment of the young ocean sediment array. (b) The noble gas abundances in young sediments as reported by [4,9,24]. KH-77-1-7 (solid gray line) shows the greatest absolute Xe enrichment of the young ocean sediment array. (c) The noble gas patterns in shales [4,10,28]. One Pleistocene claystone [10] and kerogen fractions [28] have not been plotted. (d) The noble gas abundances in shales $[4,10,28]$. (e) Noble gas patterns in cherts from sedimentary systems [30]. (f) The noble gas abundances of cherts from sedimentary systems [30].

Figure 6: (a) Noble gas patterns in obsidians and glasses [31]. (b) Noble gas concentrations in obsidians and glasses [31]. 
Figure 1

(A) Diffusive Flux

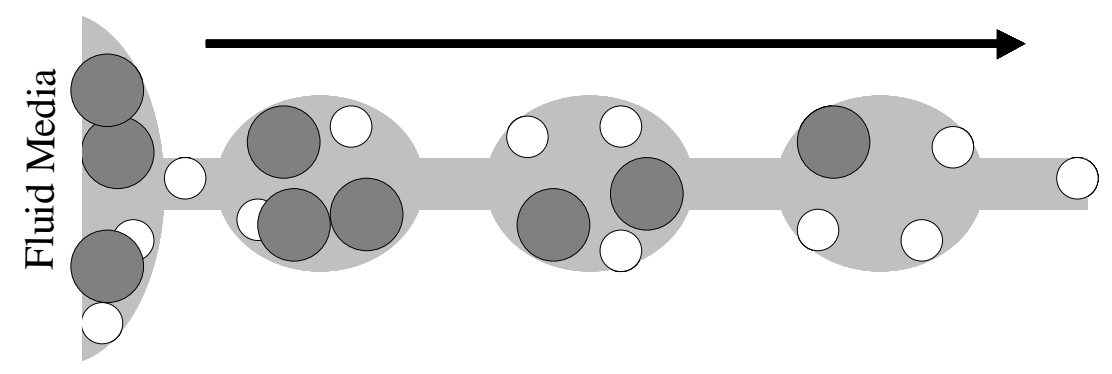

Labyrinth with Constrictions Model

for Noble Gas Adsorption on Porous Solid

(B)
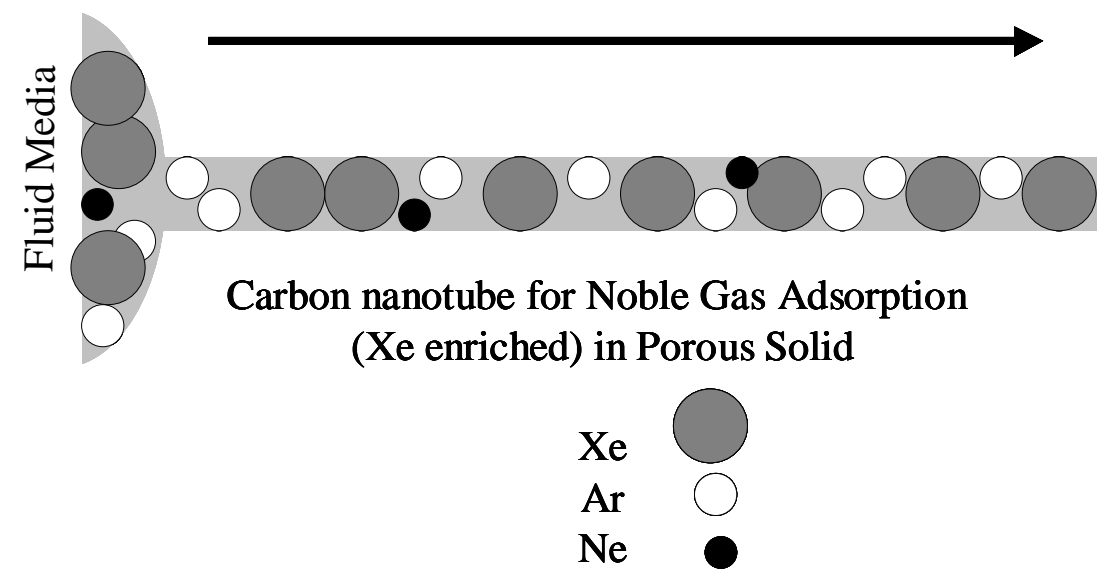
`Figure 2

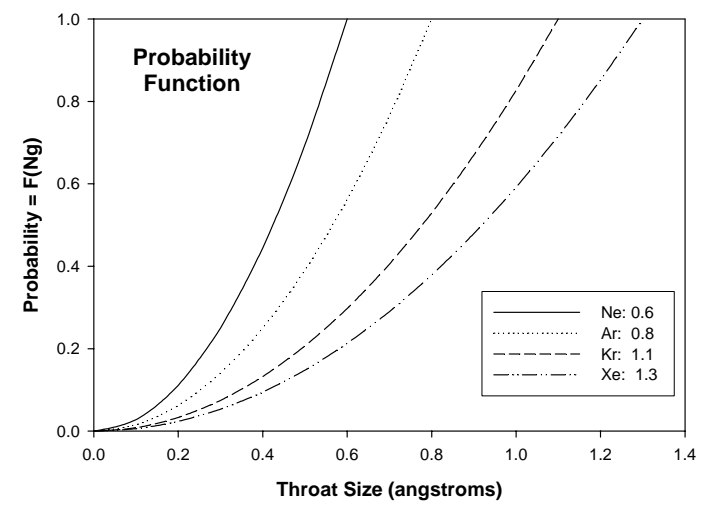


Figure 3

\section{Emptying System}
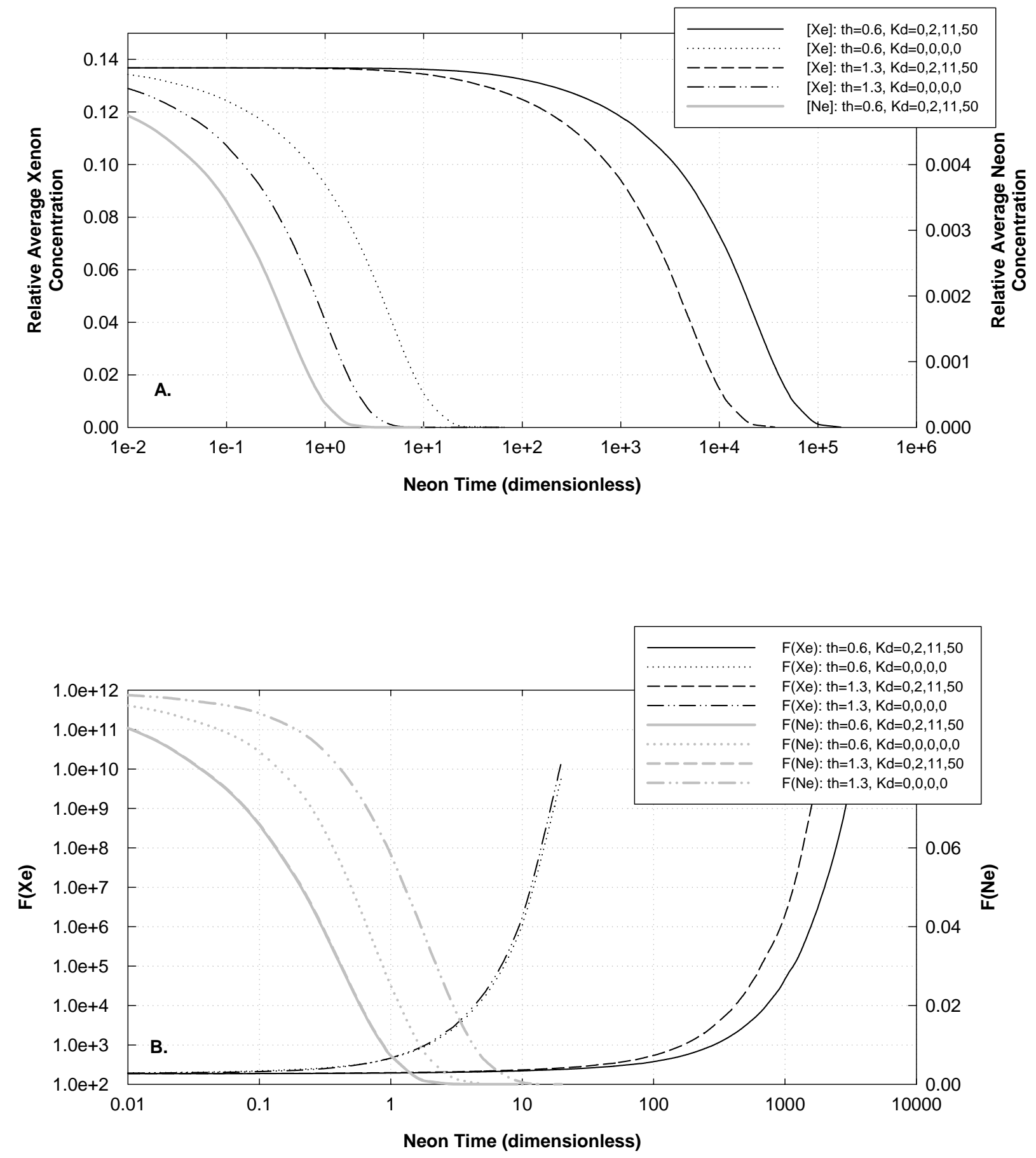
Figure 4
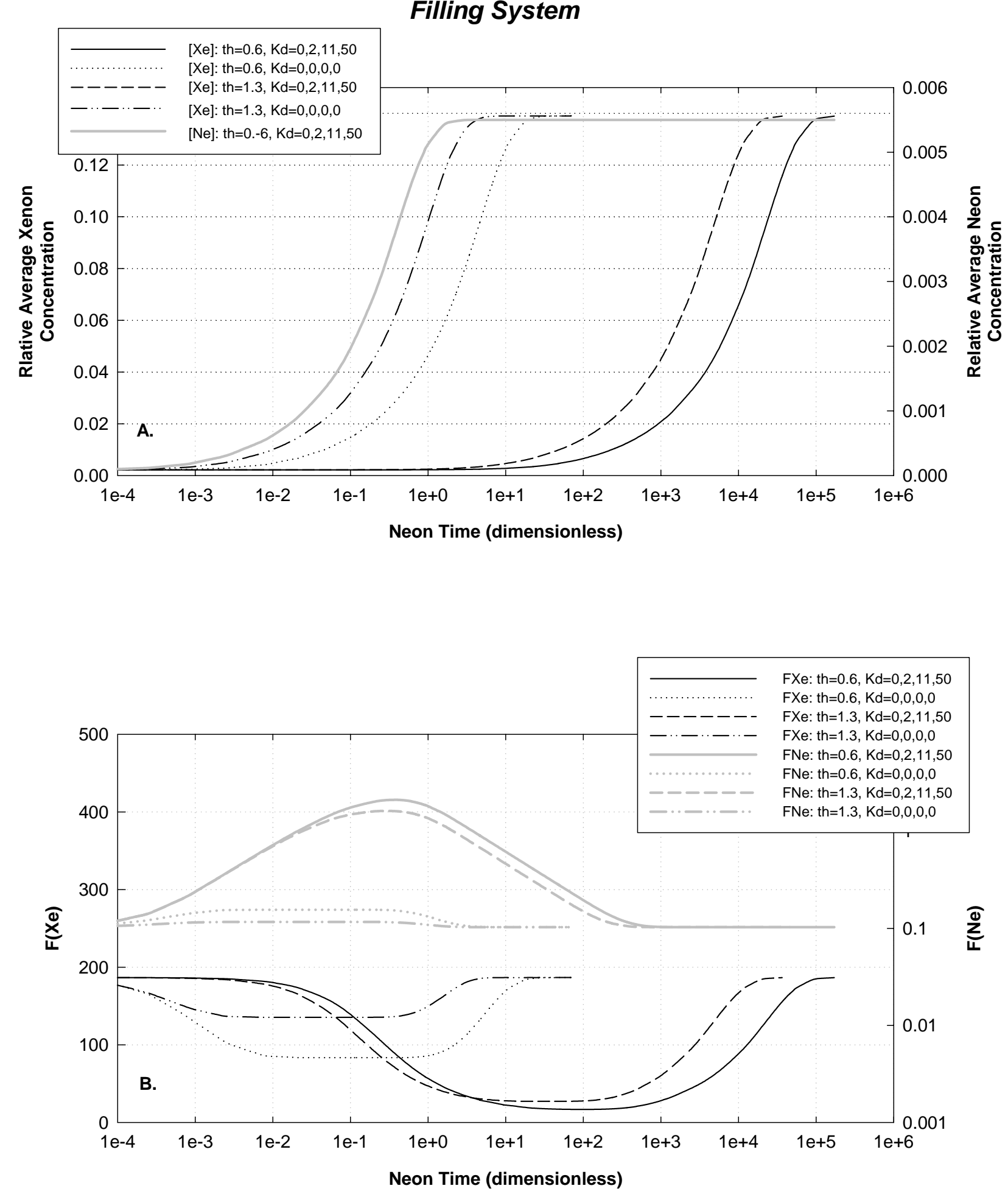

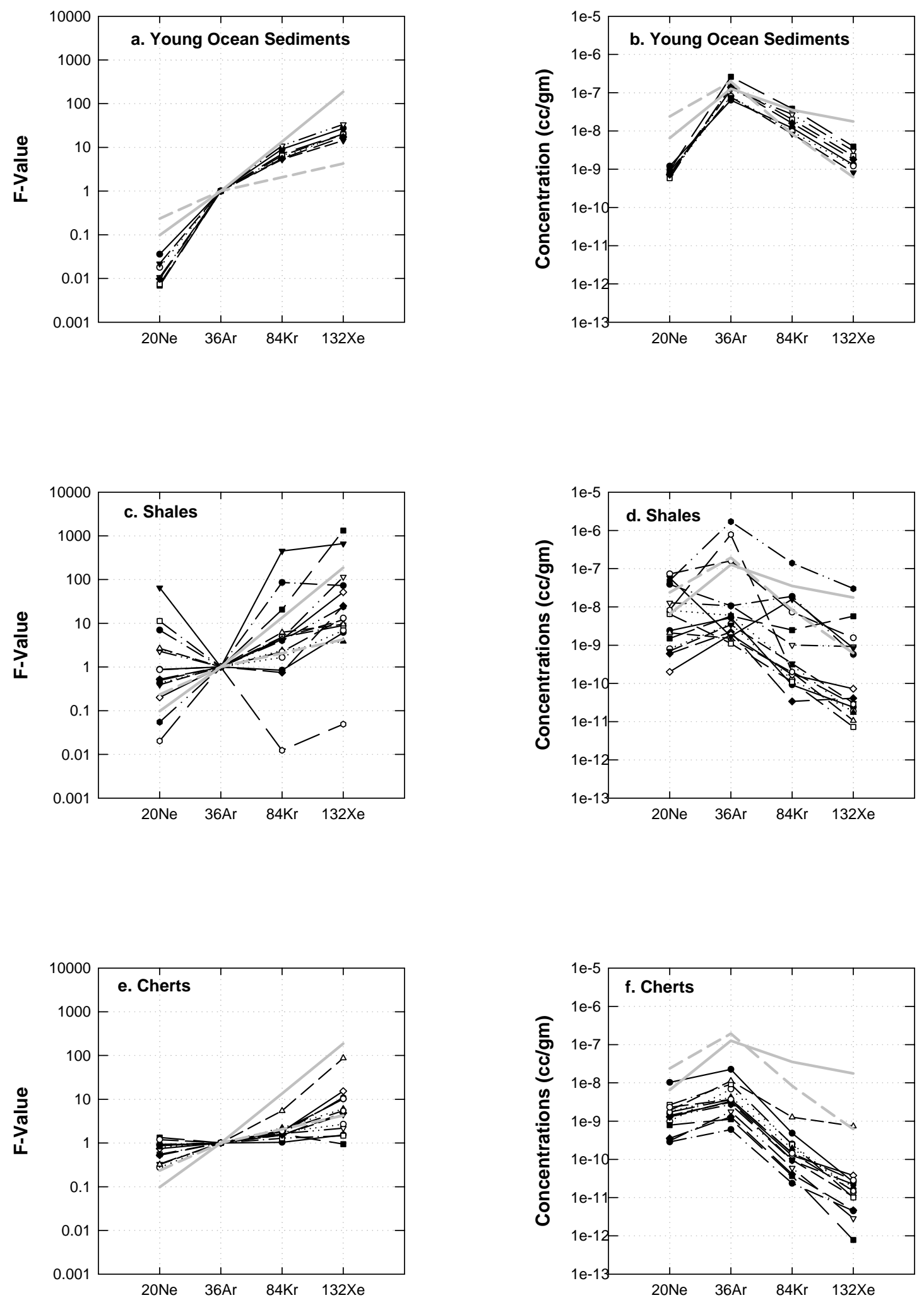

Figure 5 
Figure 6
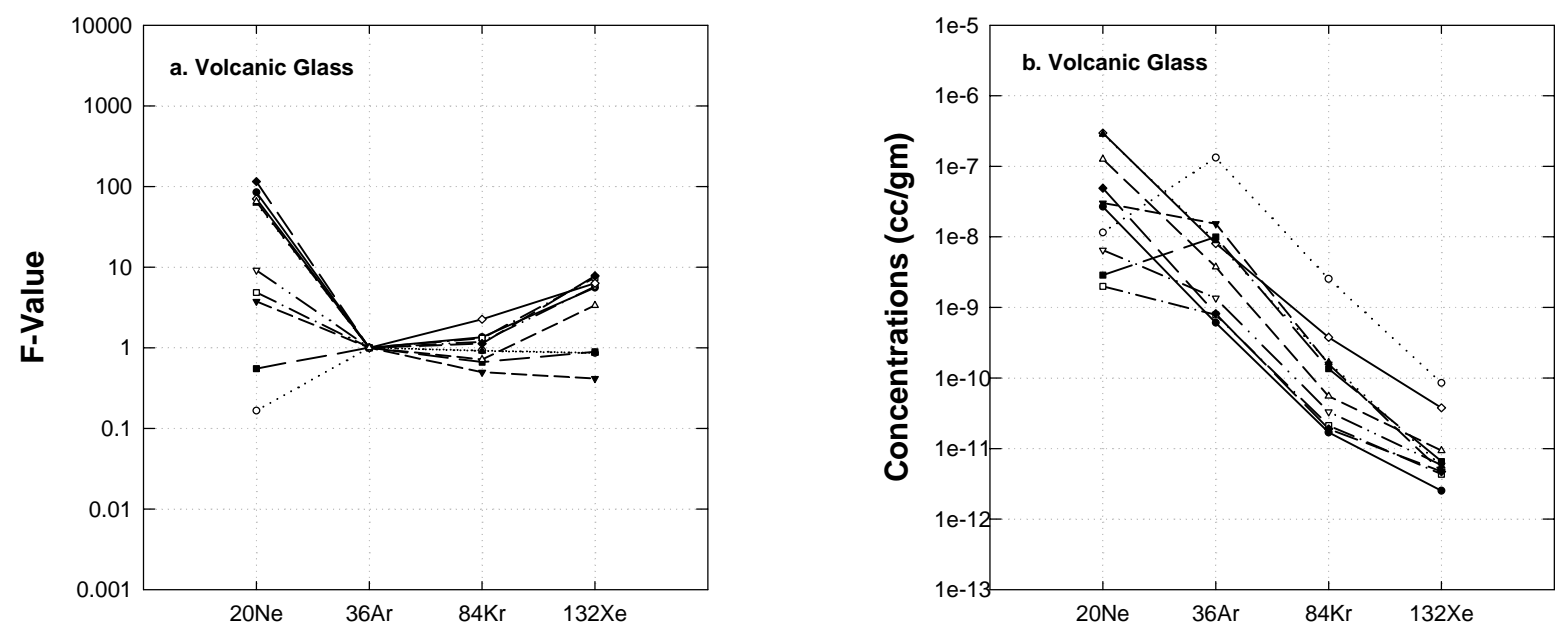